\title{
Ion implantation into ZrNb nanometric multilayers
}

\author{
Miroslav Karlík ${ }^{1,2}$, Nabil Daghbouj ${ }^{3}$, Jan Lörinčík ${ }^{4}$, Tomáš Polcar $^{3}$, Mauro Callisti $^{5}$, Vladimír Havránek $^{6}$ \\ ${ }^{1}$ Department of Physics of Materials, Faculty of Mathematics and Physics, Charles University, Ke Karlovu 5, 12116 Prague 2, \\ Czech Republic \\ ${ }^{2}$ Department of Materials, Faculty of Nuclear Sciences and Physical Engineering, Czech Technical University in Prague, Trojanova \\ 13, 12000 Prague 2, Czech Republic \\ ${ }^{3}$ Department of Control Engineering, Faculty of Electrical Engineering, Czech Technical University in Prague, Technická 2, 16000 \\ Prague 6, Czech Republic

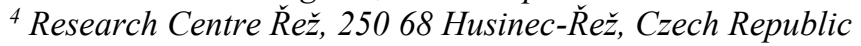 \\ ${ }_{5}^{5}$ Department of Materials Science and Metallurgy, University of Cambridge, 27 Charles Babbage Road, Cambridge, CB3 OFS, \\ United Kingdom \\ ${ }^{6}$ Nuclear Physics Institute CAS, 25068 Řež, Czech Republic
}

$\mathrm{Zr} / \mathrm{Nb}$ nanometric multilayers deposited on $\mathrm{Si}$ substrate by magnetron sputtering, having a periodicity from 6 to $167 \mathrm{~nm}$ were subjected to room temperature irradiation by carbon, silicon and copper ions. The mechanical proprieties, ion profiles, and disordering behavior have been investigated by Secondary Ion Mass Spectrometry - SIMS, nanoindentation, X-ray diffraction - XRD, and scanning transmission electron microscopy - STEM. The damaged regions are clearly visible on STEM bright field micrographs of cross-section lamellae prepared by focused ion beam technique - FIB. Damage starts from the surface side of the multilayer, and the most damaged and disordered zone is located close to the maximum ion concentration. Near the substrate, no damage was observed. The $\mathrm{C}$ and $\mathrm{Si}$ concentration profiles detected by SIMS were not affected by the nanolayers periodicity. This agrees with the Stopping and Range of Ions in Matter - SRIM software simulations. Diffraction analyses - selective area electron diffraction, and XRD suggest a structural evolution in relation to the multilayer periodicity. For the multilayer with a periodicity of $6 \mathrm{~nm}$, and $27 \mathrm{~nm}, \mathrm{Si}, \mathrm{C}$ and $\mathrm{Cu}-$ ion irradiation led to a tensile strain in $\mathrm{Nb}$ layers and compressive strain in $\mathrm{Zr}$ layers. In contrast, for periodicity higher than $27 \mathrm{~nm}$, both $\mathrm{Zr}$ and $\mathrm{Nb}$ layers are subjected to compressive out-of-plane strain.
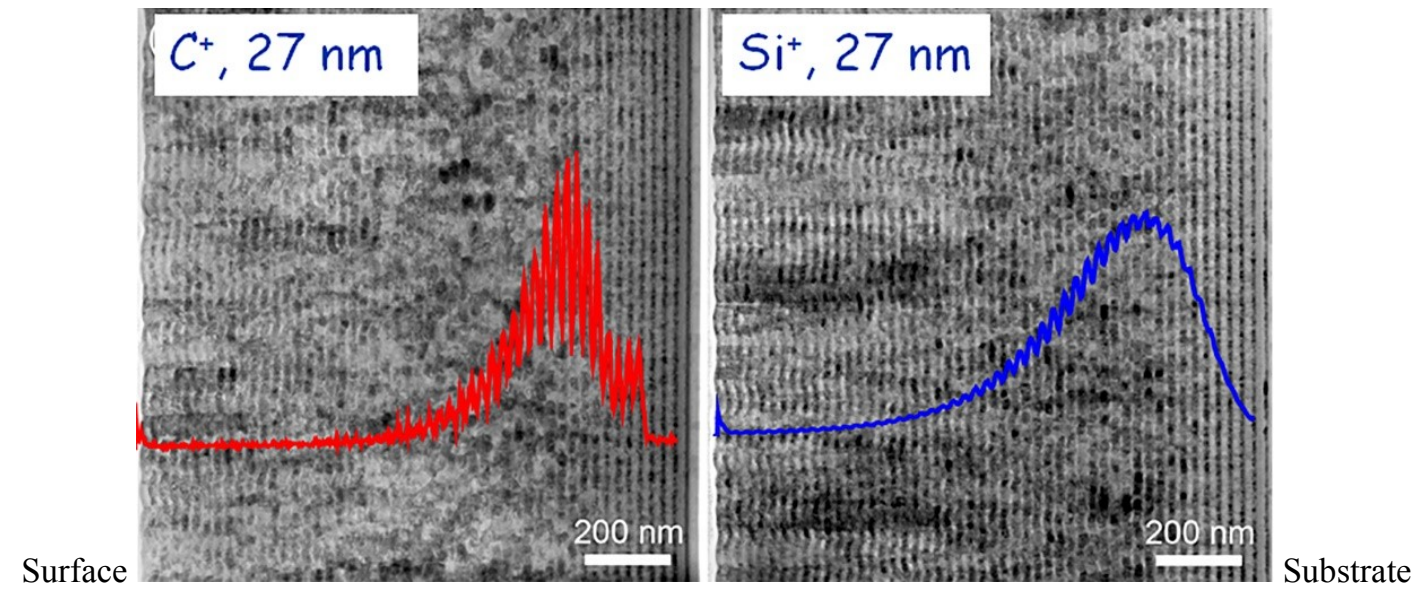

Figure 1. Highly damaged areas overlap with maximum ion concentrations detected by SIMS.

Keywords: Zr/Nb multilayers, ion irradiation, strain, XRD, SIMS 